\title{
Quantum oscillations in the SmFeAsO parent compound and superconducting $\operatorname{SmFeAs}(\mathbf{O , F})$
}

\author{
F. Caglieris,,${ }^{1,2, *}$ A. Leveratto, ${ }^{2}$ I. Pallecchi, ${ }^{2}$ F. Bernardini, ${ }^{3}$ M. Fujioka, ${ }^{4}$ Y. Takano, ${ }^{5}$ L. Repetto, ${ }^{6}$ \\ A. Jost, ${ }^{7}$ U. Zeitler, ${ }^{7}$ and M. Putti ${ }^{2,6}$ \\ ${ }^{1}$ Leibniz-Institute for Solid State and Materials Research, 01069 Dresden, Germany \\ ${ }^{2}$ SPIN-CNR, Corso Perrone 24, 16152 Genova, Italy \\ ${ }^{3}$ CNR-IOM-Cagliari and Department of Physics, University of Cagliari, 09042 Monserrato, Italy \\ ${ }^{4}$ Research Institute for Electronic Science, Hokkaido University, Sapporo, Hokkaido 001-0020, Japan \\ ${ }^{5}$ National Institute for Materials Science (NIMS), Tsukuba, Ibaraki 305-0047, Japan \\ ${ }^{6}$ Department of Physics, University of Genova, Via Dodecaneso 33, 16146 Genova, Italy \\ ${ }^{7}$ High Field Magnet Laboratory (HFML-EMFL), Radboud University, Toernooiveld 7, 6525ED Nijmegen, Netherlands
}

(Received 3 April 2017; revised manuscript received 31 July 2017; published 18 September 2017)

\begin{abstract}
By carrying out magnetotransport measurements up to $30 \mathrm{~T}$, we succeeded in detecting and analyzing quantitatively Shubnikov-de Haas oscillations in two crystals of the $R E F e A s O$ ( $R E=$ rare earth) family of iron pnictides, namely, the undoped $\mathrm{SmFeAsO}$ and the superconducting fluorine underdoped $\mathrm{SmFeAs}(\mathrm{O}, \mathrm{F})$. We combine experimental outcomes and ab initio calculations of electronic structure to get the following direct evidences: (i) the Shubnikov-de Haas oscillations are determined by a two-dimensional hole band, whose effective mass is $m^{*}=(0.50 \pm 0.1) m_{e}$ in the undoped sample and $m^{*}=(0.89 \pm 0.1) m_{e}$ in the F-doped sample, while classical magnetotransport is dominated by an electron band; (ii) in the superconducting compound we give a quantitative account of the low effectiveness of electron doping associated with fluorine substitution.
\end{abstract}

DOI: 10.1103/PhysRevB.96.104508

\section{INTRODUCTION}

Fe-based superconductors (Fe-SCs) belong to a large variety of compounds, sharing common features in terms of crystal structure, electronic structure, and magnetic ordering of the respective parent compounds. In particular, the almost nested, quasi-two-dimensional electron and hole Fermi surfaces of the parent compounds are ubiquitous and they are believed to play a crucial role in the unconventional pairing mechanism for superconductivity. In this context, extensive experimental investigation of the electronic structure of both parent and superconducting compounds across the various families of $\mathrm{Fe}-\mathrm{SCs}$ is of vital importance to understand the mechanisms of high-temperature superconductivity. Specifically, Shubnikovde Haas $(\mathrm{SdH})$ oscillation measurements are a powerful and straightforward probe of electronic parameters and Fermi surface topology, especially if complemented with $a b$ initio calculations of the band structure or compared with data from other experimental techniques. Due to the availability of millimeter-size single crystals, parent compounds of the $A \mathrm{Fe}_{2} \mathrm{As}_{2}(A=$ alkaline earth metal) family have been mostly studied in terms of $\mathrm{SdH}$ oscillations since the earliest times [1-4]. Data on superconducting members of other families are also available, namely, FeSe [5-7] and LiFeAs [8]. In the case of such superconducting samples, the large applied field must exceed the upper critical field to suppress superconductivity and allow the observation of quantum effects. In these experiments, $\mathrm{SdH}$ oscillations have been detected by different techniques, namely, as a magnetic-field-dependent change in the resonance frequency of a tunnel diode oscillator circuit [1-3,5], in magnetic torque experiments [8,3], or in transport measurements $[4,6]$. However, the agreement with band structure predicted by theory and with data from angle-resolved

*Corresponding author: f.caglieris@ifw-dresden.de photoemission spectroscopy is not always satisfying [4], and further studies would be needed in this respect. Moreover, in none of these works is the evolution of the behavior of quantum oscillations across the phase diagram spanned from the undoped parent compound towards the doped superconducting compounds ever explored. Even more importantly, on the $R E \mathrm{FeAsO}(R E=$ rare earth) family, also called FeAs-1111 family, the one with the largest transition temperature $T_{c}=$ $58 \mathrm{~K}$ at optimal doping [9], no data of $\mathrm{SdH}$ oscillations have been reported so far. In Refs. [10,11], SdH oscillations have been observed on the isostructural compound $\mathrm{LaFePO}$, which has been reported to be either nonsuperconducting [12] or superconducting with low $T_{c} \approx 6 \mathrm{~K}[10,11,13]$. However, this compound is nonmagnetic and does not exhibit any evidence of Fermi surface nesting or spin density wave transition; hence its behavior may not be representative in the context of nonconventional superconductivity of iron-based compounds. The reason for the difficulty in getting quantum oscillation data on the $R E \mathrm{Fe} A s \mathrm{O}$ family is the scarce availability of single crystals that have simultaneous high-quality transport properties and size that can be handled [14].

In this work, we aim to fill two of the above-mentioned gaps in the available literature by presenting $\mathrm{SdH}$ oscillations from transport measurements in field up to $30 \mathrm{~T}$ in two single crystals of the $R E \mathrm{Fe} A s \mathrm{O}$ family, namely, undoped $\mathrm{SmFeAsO}$ and fluorine underdoped $\operatorname{SmFeAs}(\mathrm{O}, \mathrm{F})$, the latter exhibiting signatures of both magnetic ordering and a superconducting transition. Our data confirm the two-dimensional character of electronic structure and the multiband scenario, both predicted by our density functional theory (DFT) calculations. Moreover, on the basis of the information on band structure and Fermi surface shape obtained from DFT calculations, we fit our SdH oscillation data in a single-band scenario and extract relevant electronic parameters for the hole band in the two samples, such as effective masses, carrier concentrations, and scattering times. 


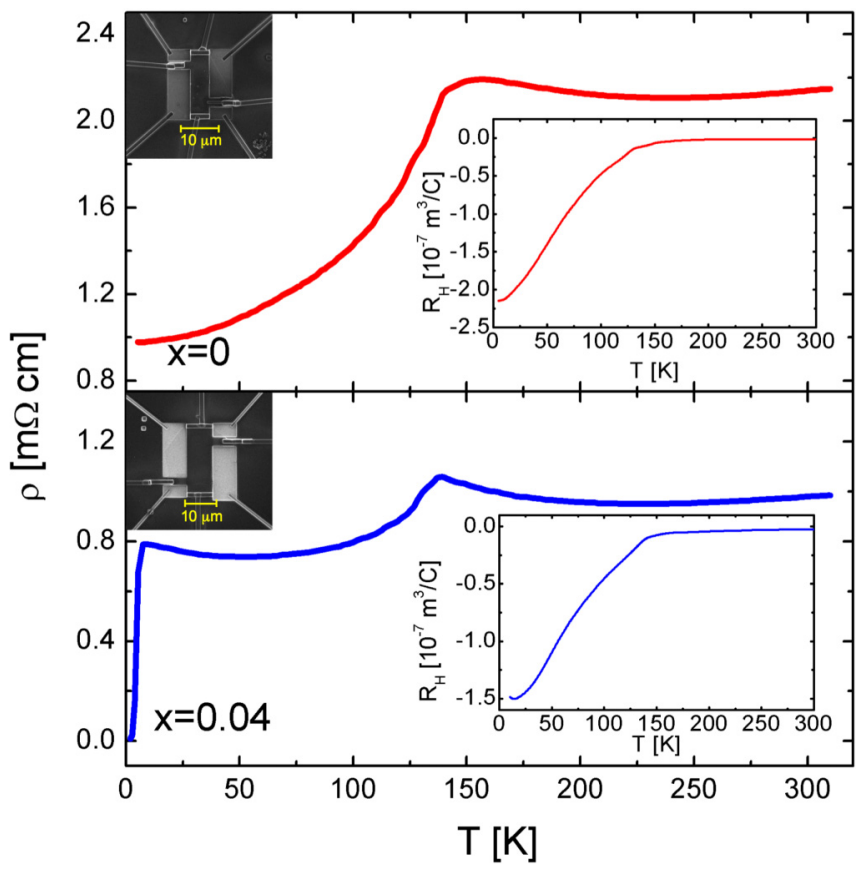

FIG. 1. Main panels: Resistivity versus temperature curves measured in the $\mathrm{SmFeAsO}$ (top) and $\mathrm{SmFeAsO}_{0.96} \mathrm{~F}_{0.04}$ (bottom) crystals. Top-left insets: SEM images of the crystals with tungsten electrical contacts; the scale bar is $10 \mu \mathrm{m}$. Right insets: Hall resistance $R_{H}$ versus temperature curves.

\section{EXPERIMENTAL DETAILS}

Our $\mathrm{SmFeAsO}$ and $\mathrm{SmFeAs}(\mathrm{O}, \mathrm{F})$ single-crystal samples were prepared by flux method using $\mathrm{CsCl}$ as described in Ref. [15]. The in-plane (parallel to FeAs planes) crystal sizes are $5.1 \times 9.8$ and $6.8 \times 12.0 \mu \mathrm{m}^{2}$, respectively, which is large enough to allow the fabrication of tungsten electrodes by focused ion beam (FIB) for transport measurements, as shown in the scanning electron microscope (SEM) images in the insets of Fig. 1. Magnetotransport measurements were carried out in magnetic fields up to $30 \mathrm{~T}$, applied perpendicular to the sample surface (parallel to the $c$ axis), and temperatures down to $380 \mathrm{mK}$. Measurements of resistivity and Hall effect up to room temperature were performed in a commercial PPMS (Physical Properties Measurement System, by Quantum Design) in fields up to $9 \mathrm{~T}$.

\section{RESULTS AND DISCUSSION}

\section{A. Experimental data and analysis}

In Fig. 1, the temperature dependences of the resistivity and the Hall resistance of each sample are shown. The undoped sample (left) exhibits a sharp signature of the structural/magnetic transition at $T_{N} \approx 136 \mathrm{~K}$ (calculated from the maximum of the derivative of the resistivity), below which metallic behavior is observed. The underdoped sample (right) exhibits as well a signature of the structural/magnetic transition, whose sharpness points to the high quality of the sample but at a smaller temperature $T_{N} \approx 127 \mathrm{~K}$, and a high-temperature resistivity value smaller by a factor of 2 . A superconducting transition is seen at $T_{c} \approx 6 \mathrm{~K}$ (calculated at the onset of the resistivity drop, while the zero resistivity state is not completely reached even at the lowest measuring temperatures). This superconducting phase lies in the underdoped regime and has an upper critical field $\mu_{0} H_{c 2} \approx 12 \mathrm{~T}$ at $0.3 \mathrm{~K}$, as estimated from the change of concavity in the resistance versus field curve (see Supplemental Material [16] for details). The coherence length, which can be extracted as $\xi_{0}=\sqrt{\Phi_{0} / 2 \pi \mu_{0} H_{c 2}} \approx 5 \mathrm{~nm}$, is in trend with values of $\xi_{0} \approx 3 \mathrm{~nm}$ for $T_{c}=33 \mathrm{~K}$ and $\xi_{0} \approx 2 \mathrm{~nm}$ for $T_{c}=52 \mathrm{~K}$ [17]. This suggests the bulk nature of superconductivity. For a comparison of the effect of nominal fluorine doping of $4 \%$ on $T_{N}$ and $T_{c}$ as extracted from transport measurements, we note that available literature phase diagrams are all built from data on polycrystalline samples and show somewhat scattered values of $T_{N}$ and $T_{c}$ as a function of fluorine doping [18-21]. Thus they may not be an ideal reference to our case; however, our data are in fair agreement with Ref. [18].

The magnitude of the upper critical field, $H_{c 2} \approx 12 \mathrm{~T}$ at $0.3 \mathrm{~K}$ (see Supplemental Material [16] for details), is small enough to be suppressed by the applied field, allowing quantum oscillation measurements. The temperature dependence of the Hall resistance $R_{H}$, also shown in Fig. 1, indicates that for both samples the structural/magnetic transition triggers a condensation of charge carriers, likely related to the opening of a spin density wave gap, determining an increase in the magnitude of $R_{H}$ down to low temperatures. At low temperatures, the negative sign of $R_{H}$ indicates that an electron-type band dominates transport in both samples, and the smaller magnitude of $R_{H}$ in the doped sample is consistent with the introduction of $n$-type carriers by fluorine doping. In the higher temperature regime above $T_{N}, R_{H}$ changes from values around $-1 \times 10^{-9} \mathrm{~m}^{3} / \mathrm{C}$ in the undoped sample to values around $-3 \times 10^{-9} \mathrm{~m}^{3} / \mathrm{C}$ in the doped sample. Possibly, the effect of $n$-type doping in this temperature range can be seen as a departure from electron-hole compensation towards electron predominance.

Magnetoresistance measurements were performed up to $30 \mathrm{~T}$ with field perpendicular and parallel to the FeAs plane. (Raw magnetoresistance data are presented in the Supplemental Material [16].) SdH oscillations are only observed in perpendicular field, which points to the two-dimensional character of the Fermi surface in these compounds. In contrast, in 122 parent compounds, quantum oscillations have been observed also in parallel field [2-4], indicating a more threedimensional character. To evidence oscillations, from our raw magnetoresistance data, a nonoscillating magnetoresistance background of the form $\rho_{\mathrm{bg}}(B)=\rho_{0}+\rho_{1} B+\rho_{2} B$ [2] was subtracted, where $\rho_{1}$ and $\rho_{2}$ are fitting constants representing the linear Abrikosov quantum magnetoresistivity and quadratic cyclotronic magnetoresistivity.

In Fig. 2, we present two sets of SdH magnetoresistance oscillations $\Delta \rho=\left(\rho-\rho_{\mathrm{bg}}\right) / \rho_{0}$ measured at different temperatures in the two samples. As expected, the amplitude of the oscillations is exponentially damped with increasing temperature and with increasing inverse field $1 / B$, as predicted by the Lifshitz-Kosevic (L-K) formalism [22], described in the Supplemental Material [16]. We first analyzed our experimental curves by performing the fast Fourier transform (FFT). The results for the undoped and the doped samples are shown in the insets of Figs. 3(a) and 3(b), respectively: in both 


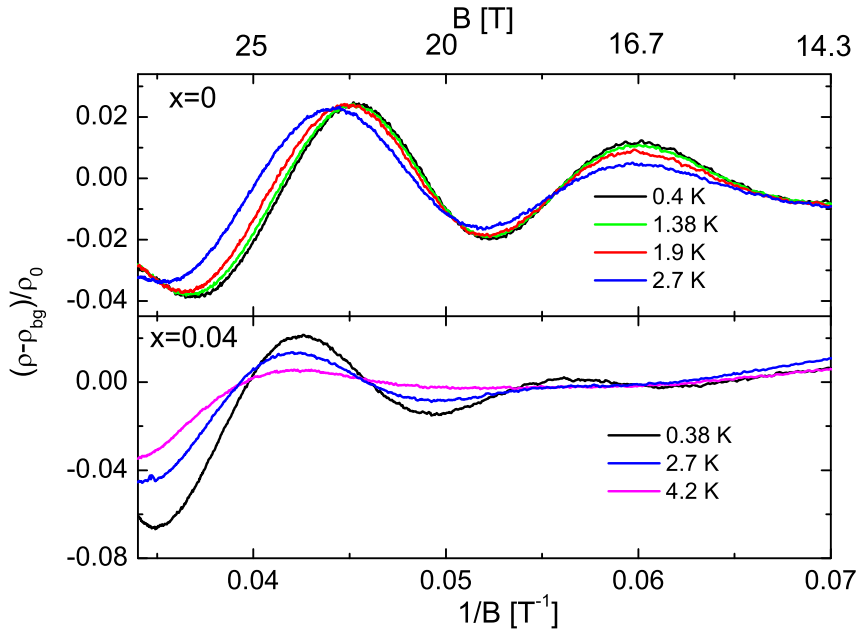

FIG. 2. Normalized magnetoresistivity versus inverse field (bottom axis) curves measured in the SmFeAsO (top) and $\mathrm{SmFeAsO} \mathrm{A}_{0.96} \mathrm{~F}_{0.04}$ (bottom) crystals. In particular, the nonoscillating background magnetoresistance $\rho_{\mathrm{bg}}$ is subtracted from the raw resistivity data $\rho$ and the result is normalized to the zero field resistivity $\rho_{0}$.

cases a main peak centered at a frequency $F_{B}=(65 \pm 6) \mathrm{T}$ is found. According to the relationship $F_{B}=2 e / h n_{2 \mathrm{D}}$, where $h$ is the Plank constant, $e$ the electron charge, and $n_{2 \mathrm{D}}$ the two-dimensional carrier concentration $\left(n=n_{2 \mathrm{D}} / c\right.$, with $c$ the out-of-plane lattice parameter), these frequencies correspond to volume carrier concentrations $n=(3.9 \pm 0.3) \times 10^{25} \mathrm{~m}^{-3}$.

In addition, we obtain the effective masses $m^{*}$ of carriers in these bands associated to the frequency $F_{B}$, by fitting the temperature evolution of the FFT peak amplitudes with the function $\chi / \sinh (\chi)$, where $\chi=2 \pi^{2} k_{\mathrm{B}} m^{*} T /(e B)$, and $k_{\mathrm{B}}$ is the Boltzmann constant. The outcomes of this analysis are $m^{*}=(0.50 \pm 0.1) m_{e}$ and $m^{*}=(0.89 \pm 0.1) m_{e}$ for the undoped and doped samples, respectively.

Since only one main frequency can be extracted from our $\mathrm{SdH}$ oscillations, we fit the experimental data with the complete L-K formula at $T=0.4 \mathrm{~K}$ using the FFT frequency as a fixed input parameter. As shown in Fig. 4, we obtain a very good agreement between the data and the model. This fitting procedure gives as output parameters both the effective masses $m^{*}$ and the scattering times $\tau$. In particular, for the parent compound $\mathrm{SmFeAsO}$ we obtain $m^{*}=0.53 m_{e}$ and $\tau=$ $1.21 \times 10^{-13} \mathrm{~s}$, while for the doped sample $\mathrm{SmFeAsO}_{0.96} \mathrm{~F}_{0.04}$ we obtain $m^{*}=1.00 m_{e}$ and $\tau=1.53 \times 10^{-13} \mathrm{~s}$. The effective masses extracted from the fit with the complete L-K formula are pretty consistent with the values obtained from the analysis of temperature dependence of the FFT peak.

\section{B. Theoretical calculations}

We now turn to the results of DFT calculations. DFT calculations are performed in the antiferromagnetic state (see Supplemental Material for details [16]). In Fig. 5 (inset) we show a perspective view of the Fermi surface (FS), with one hole sheet (red) and two electron sheets (blue). The hole FS is a nearly perfect cylinder with negligible dispersion along $k_{z}$. The electron FSs are two weakly bent cylinders with a slightly elliptical cross section. The shape of both hole and electron FSs is in good agreement with DFT calculations performed in Ref. [23] on LaFeAsO. The ratio of the areas of the two extremal cross sections is close to unity, 1.4 and 1.7 for the holes and electrons, respectively, confirming their almost cylindrical nature. Stoichiometric $\mathrm{SmFeAsO}$ is a perfectly compensated semimetal. Our calculations confirm this finding. Indeed, the volume inside each electron FS is exactly one-half of the corresponding volume of the hole FS. The compensated nature of $\mathrm{SmFeAsO}$, together with the 2D dispersion of the bands at $E_{F}$, brings us to the important result that the average cross-sectional area of the electron FS must be half of the corresponding one for the hole. This latter result is valid only for the undoped compound. Upon F doping, the balance of electrons and holes is broken and electrons are added into the system. Given the low doping concentrations studied here, we use a rigid-band approach to simulate the effect of F doping on the FS's shapes and sizes. In Fig. 5, we show a plot of the calculated oscillation frequencies $F_{B}$ as a function of electron doping in $\mathrm{SmFeAsO}$. We see that for the undoped compound, $F_{B}$ values are 30 and $60 \mathrm{~T}$ for electrons and holes, respectively. Remarkably, the frequency associated to holes corresponds to our experimental values extracted from quantum oscillations in both the undoped and doped samples, which makes it plausible to associate the experimental quantum oscillations to this hole band. (a)

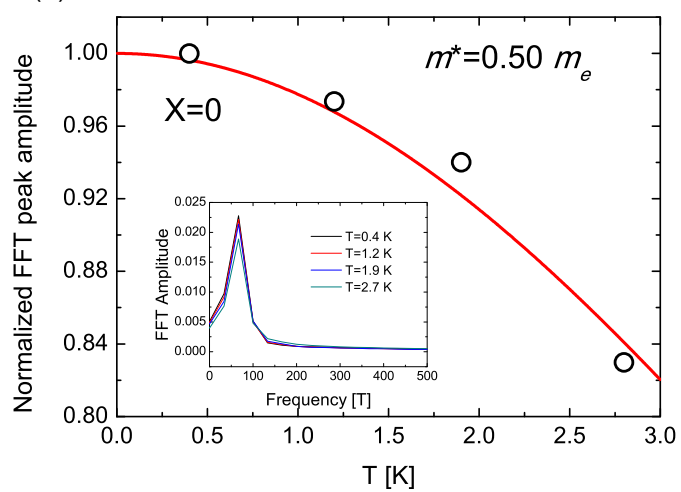

(b)

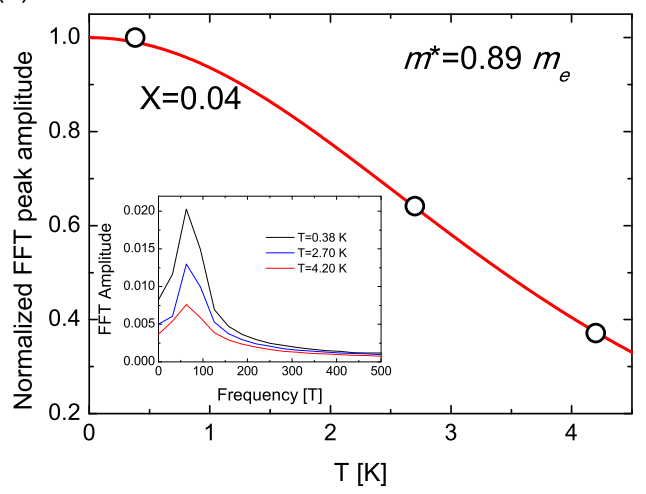

FIG. 3. Temperature evolution of the FFT peak amplitudes for the $\mathrm{SmFeAsO}$ (a) and $\mathrm{SmFeAsO}_{0.96} \mathrm{~F}_{0.04}$ (b) crystals. Continuous lines are fitting functions (see text). 


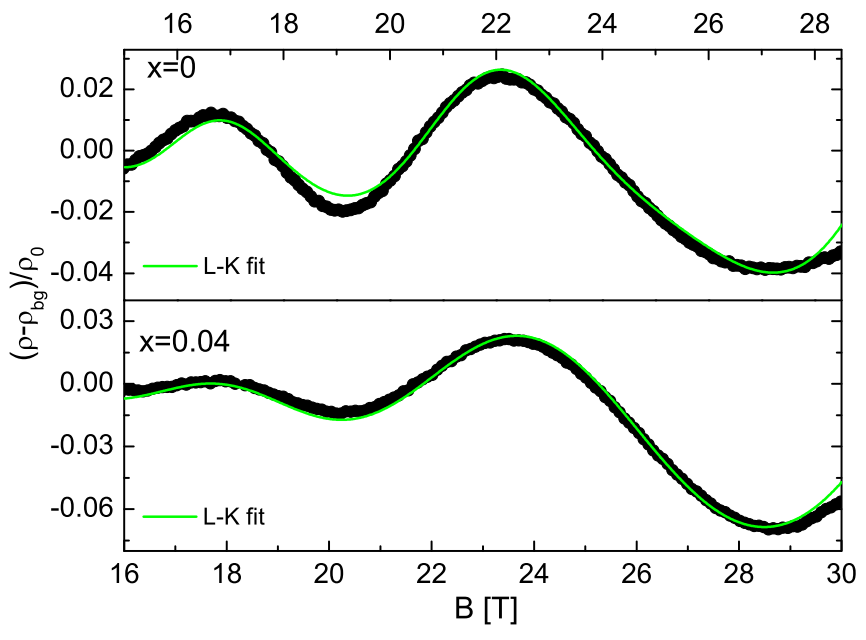

FIG. 4. Oscillating magnetoresistivity versus field curves measured in the $\mathrm{SmFeAsO}_{1-x} \mathrm{~F}_{x}$ with $x=0$ (top) and $x=0.04$ (bottom) crystals. Single-band L-K fittings are shown as continuous lines.

DFT calculations indicate the existence of two types of carriers whose densities should be balanced in the undoped sample. The multiband description is also necessary to account for the behavior of the Hall effect data (insets of Fig. 1), whose temperature dependence is not compatible with a single-band scenario [24]. Moreover, the Hall coefficient is negative at low temperature, suggesting that it is dominated by an electron band, opposite to $\mathrm{SdH}$ oscillations that we associate to the hole band. In order to account for this phenomenology, we can simply consider the two-band model for the Hall coefficient $R_{h}=\left(n_{h} \mu_{h}^{2}-n_{e} \mu_{e}^{2}\right) /\left[e\left(n_{e} \mu_{e}+n_{h} \mu_{h}\right)^{2}\right]$, where $n_{e / h}$ and $\mu_{e / h}$ are the electron/hole densities and mobilities, respectively. If we assume $n_{e}=n_{h}$ for the undoped sample, according to the compensation predicted by DFT calculations, it is straightforwardly seen that any $\mu_{e}$ larger than $\mu_{h}$ yields a negative Hall coefficient. Moreover, if we use the scattering time and the effective mass of the

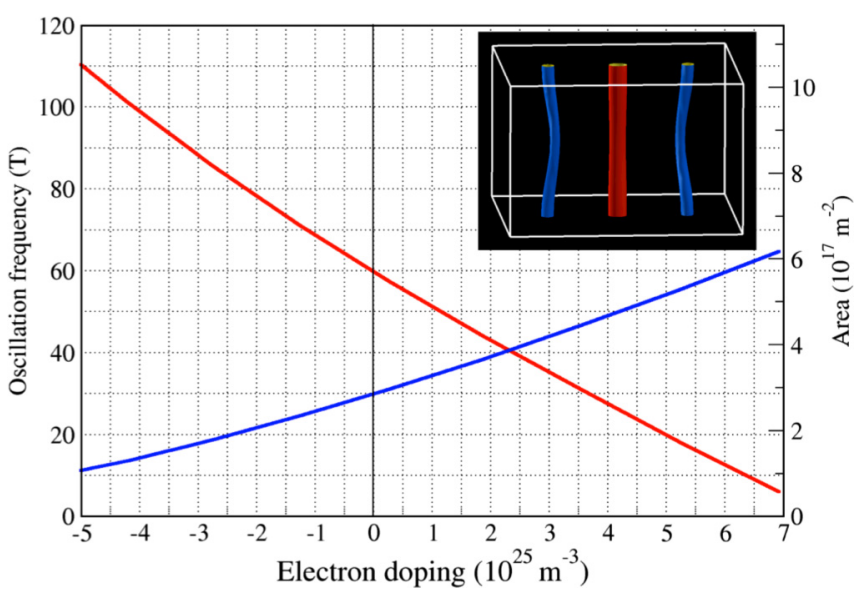

FIG. 5. Main panel: Computed value for the $\mathrm{SdH}$ oscillation frequency as a function of doping (blue for electrons and red for holes). Positive values correspond to $n$-type doping. On the right $Y$ axis the cross-section areas are given. Inset: View of the Fermi surfaces of $\mathrm{SmFeAsO}$ inside the first Brillouin zone of the four formula unit cell. hole band obtained from quantum oscillations to evaluate the hole mobility $\mu_{h}$ and we assume the same $n$ value for both the bands as obtained from the oscillation frequencies, we find that the so-calculated $R_{h}$ well approximates the experimental value of low-temperature Hall coefficient in the limit $\mu_{e} \gg \mu_{h}$, where $R_{h} \approx\left(-e n_{e}\right)^{-1}=-(1.6 \pm 0.2) \times 10^{-7} \mathrm{~m}^{3} / \mathrm{C}$. Hence, by combining our measurements with DFT calculations, we obtain that a high-mobility electron band should exist to explain transport measurements. Remarkably, this finding agrees with the presence of high-mobility electron-type Dirac cones in the band structures of these parent compounds, predicted by our $a b$ initio calculations (see Supplemental Material [16] for details), and also theoretically predicted and confirmed by other experimental techniques in literature both in 1111 [25,26] and in 122 [27] families. According to calculations, these linear dispersing bands are associated to cylindrical electronic FS, whose cross section is around half of the hole FS (see inset of Fig. 5). This implies that the period of the oscillations should be twice as much as the period of the hole band, which requires higher magnetic fields than those reached in this experiment to be unambiguously detected.

Let us now turn our attention to the doped sample. According to DFT calculations, in the rigid-band approach, the doping increases (decreases) the electron (hole) $F_{B}$, so that above a $2.3 \times 10^{25} \mathrm{~m}^{-3}$ electron doping concentration, the electrons should have a frequency higher than that of the holes (see Fig. 5). On the contrary, the frequency of the quantum oscillations that we observe in the doped sample is almost the same as that of the parent compound, as if the carrier density of the hole band was not affected by the substitution. Hence, we gather that $\mathrm{F}$ substitution is less effective than what is predicted by the application of the rigid-band model to the band structure obtained by DFT calculations. The explanation for this seeming discrepancy between theory and experiment is that F does not behave as a shallow donor but it is actually a deep impurity. Hence, the band structure is altered by the introduction of $\mathrm{F}$ impurities across a wide spectrum of energies, so that on one hand the rigid-band approximation still works, because in proximity of the Fermi level the band changes are not significant, and on the other hand the change in the band filling is less than nominal, that is, less than one electron per F. This scenario is confirmed by band structure calculations on the SmFeAsF compound (see Supplemental Material [16] for details).

\section{CONCLUSIONS}

In conclusion, we measured the magnetotransport properties of two high-quality $\mathrm{SmFeAsO}_{1-x} \mathrm{~F}_{x}(x=0 ; 0.04)$ single crystals of several microns size, in magnetic fields up to $30 \mathrm{~T}$ and temperatures down to $380 \mathrm{mK}$. Two-dimensional Shubnikov-de Haas oscillations are observed in both samples, remarkably also in the superconducting fluorine underdoped sample $\left(T_{c}=6 \mathrm{~K}\right)$ for fields above $16 \mathrm{~T}$ well exceeding the upper critical field. We analyze quantitatively the $\mathrm{SdH}$ oscillation curves within the L-K formalism. From the comparison with our $a b$ initio calculations of electronic structure and Fermi surfaces, we identify the oscillating band with the hole sheet of the Fermi surface for both samples. By fitting the temperature dependence of the oscillation amplitudes, 
we extract effective mass values $m^{*}=(0.50 \pm 0.1) m_{e}$ and $m^{*}=(0.89 \pm 0.1) m_{e}$ for the undoped and doped samples, respectively. The comparison of oscillation frequencies and low-temperature Hall effect data in the two samples suggest that $\mathrm{F}$ substitution is significantly less effective than what is predicted by DFT calculations in terms of band filling. This evidence of the low effectiveness of fluorine doping establishes the correct scale along the doping axis of the phase diagram of these compounds.

\section{ACKNOWLEDGMENTS}

We acknowledge the support of FP7 European project SUPER-IRON (Grant Agreement No. 283204) and the support of the HFML-RU/FOM, member of the European Magnetic Field Laboratory (EMFL). F.B. acknowledges partial support from the project "Multiphysics Approach to Thermoelectricity," funded by Fondazione Sardegna (2017), and the PRID 2016 project funded by the University of Cagliari.
[1] S. E. Sebastian, J. Gillett, N. Harrison, P. H. C. Lau, D. J. Singh, C. H. Mielke, and G. G. Lonzarich, J. Phys.: Condens. Matter 20, 422203 (2008).

[2] N. Harrison, R. D. McDonald, C. H. Mielke, E. D. Bauer, F. Ronning, and J. D. Thompson, J. Phys.: Condens. Matter 21, 322202 (2009).

[3] J. G. Analytis, R. D. McDonald, J.-H. Chu, S. C. Riggs, A. F. Bangura, C. Kucharczyk, M. Johannes, and I. R. Fisher, Phys. Rev. B 80, 064507 (2009).

[4] T. Terashima, N. Kurita, M. Tomita, K. Kihou, C.-H. Lee, Y. Tomioka, T. Ito, A. Iyo, H. Eisaki, T. Liang, M. Nakajima, S. Ishida, S.-i. Uchida, H. Harima, and S. Uji, Phys. Rev. Lett. 107, 176402 (2011).

[5] A. Audouard, F. Duc, L. Drigo, P. Toulemonde, S. Karlsson, P. Strobel, and A. Sulpice, Europhys. Lett. 109, 27003 (2009).

[6] T. Terashima, N. Kikugawa, A. Kiswandhi, E.-S. Choi, J. S. Brooks, S. Kasahara, T. Watashige, H. Ikeda, T. Shibauchi, Y. Matsuda, T. Wolf, A. E. Böhmer, F. Hardy, C. Meingast, H. v. Löhneysen, M.-T. Suzuki, R. Arita, and S. Uji, Phys. Rev. B 90, 144517 (2014).

[7] M. D. Watson, T. K. Kim, A. A. Haghighirad, N. R. Davies, A. McCollam, A. Narayanan, S. F. Blake, Y. L. Chen, S. Ghannadzadeh, A. J. Schofield, M. Hoesch, C. Meingast, T. Wolf, and A. I. Coldea, Phys. Rev. B 91, 155106 (2015).

[8] C. Putzke, A. I. Coldea, I. Guillamón, D. Vignolles, A. McCollam, D. LeBoeuf, M. D. Watson, I. I. Mazin, S. Kasahara, T. Terashima, T. Shibauchi, Y. Matsuda, and A. Carrington, Phys. Rev. Lett. 108, 047002 (2012).

[9] M. Fujioka, S. J. Denholme, T. Ozaki, H. Okazaki, K. Deguchi, D. Demura, H. Hara, T. Watanabe, H. Takeya, T. Yamaguchi, H. Kumakura, and Y. Takano, Supercond. Sci. Technol. 26, 085023 (2013)

[10] A. Carrington, A. I. Coldea, J. D. Fletcher, N. E. Hussey, C. M. J. Andrew, A. F. Bangura, J. G. Analytis, J.-H. Chu, A. S. Erickson, I. R. Fisher, and R. D. McDonald, Physica C 469, 459 (2009).

[11] A. I. Coldea, J. D. Fletcher, A. Carrington, J. G. Analytis, A. F. Bangura, J.-H. Chu, A. S. Erickson, I. R. Fisher, N. E. Hussey, and R. D. McDonald, Phys. Rev. Lett 101, 216402 (2008).

[12] T. M. McQueen, M. Regulacio, A. J. Williams, Q. Huang, J. W. Lynn, Y. S. Hor, D. V. West, M. A. Green, and R. J. Cava, Phys. Rev. B 78, 024521 (2008).
[13] J. G. Analytis, J.-H. Chu, A. S. Erickson, C. Kucharczyk, A. Serafin, A. Carrington, C. Cox, S. M. Kauzlarich, H. Hope, and I. R. Fisher, arXiv:0810.5368.

[14] C. A. McElroy, J. J. Hamlin, B. D. White, M. A. McGuire, B. C. Sales, and M. B. Maple, Phys. Rev. B 88, 134513 (2013).

[15] M. Fujioka, S. J. Denholme, M. Tanaka, H. Takeya, T. Yamaguchi, and Y. Takano, Appl. Phys. Lett. 105, 102602 (2014).

[16] See Supplemental Material at http://link.aps.org/supplemental/ 10.1103/PhysRevB.96.104508 for (i) raw magnetoresistivity data in parallel and perpendicular field, (ii) details on LifshitzKosevic fit of magnetoresistivity data, (iii) details and additional results of $a b$ initio calculations of the electronic structure.

[17] I. Pallecchi, C. Fanciulli, M. Tropeano, A. Palenzona, M Ferretti, A. Malagoli, A. Martinelli, I. Sheikin, M. Putti, and C. Ferdeghini, Phys. Rev. B 79, 104515 (2009).

[18] Y. Kamihara, T. Nomura, M. Hirano, J. E. Kim, K. Kato, M. Takata, Y. Kobayashi, S. Kitao, S. Higashitaniguchi, Y. Yoda, M. Seto, and H. Hosono, New J. Phys. 12, 033005 (2010).

[19] S. Sanna, R. De Renzi, G. Lamura, C. Ferdeghini, A. Palenzona, M. Putti, M. Tropeano, and T. Shiroka, Phys. Rev. B 80, 052503 (2009).

[20] C. Hess, A. Kondrat, A. Narduzzo, J. Hamann-Borrero, R. Klingeler, J. Werner, G. Behr, and B. Büchner, Europhys. Lett. 87, 17005 (2009).

[21] A. Martinelli, F. Bernardini, and S. Massidda, C. R. Phys. 17, 5 (2016).

[22] D. Shoenberg, Magnetic Oscillations in Metals (Cambridge University Press, Cambridge, UK, 1984).

[23] Z. P. Yin, S. Lebégue, M. J. Han, B. P. Neal, S.Y. Savrasov, and W. E. Pickett, Phys. Rev. Lett. 101, 047001 (2008).

[24] S. C. Riggs, R. D. McDonald, J. B. Kemper, Z. Stegen, G. S. Boebinger, F. F. Balakirev, Y. Kohama, A. Migliori, H. Chen, R. H. Liu, and X. H. Chen, J. Phys.: Condens. Matter 21, 412201 (2009).

[25] I. Pallecchi, F. Bernardini, M. Tropeano, A. Palenzona, A. Martinelli, C. Ferdeghini, M. Vignolo, S. Massidda, and M. Putti, Phys. Rev. B 84, 134524 (2011).

[26] I. Pallecchi, F. Bernardini, F. Caglieris, A. Palenzona, S. Massidda, and M. Putti, Eur. Phys. J. B 86, 338 (2013).

[27] K. K. Huynh, Y. Tanabe, and K. Tanigaki, Phys. Rev. Lett. 106, 217004 (2011). 